\title{
Diagnóstico laboratorial das leucemias mielóides agudas
}

\author{
Laboratory diagnosis of acute myeloid leukemias
}

Graziele C. da Silva1; Diogo A. Pilger 2; Simone M. de Castro3; Sandrine C. Wagner4

\begin{tabular}{|c|c|}
\hline uni & \\
\hline $\begin{array}{l}\text { Leucemia mielóide aguda } \\
\text { Citoquímica } \\
\text { Imunofenotipagem } \\
\text { Citogenética } \\
\text { Genética molecular }\end{array}$ & $\begin{array}{l}\text { As leucemias agudas caracterizam-se pela proliferação clonal e pelo bloqueio maturativo das células } \\
\text { hematopoéticas, com substituição difusa da medula óssea por células neoplásicas. A leucemia mielóide } \\
\text { aguda (LMA) é um grupo heterogêneo de doenças clonais do tecido hematopoético, que acomete } \\
\text { predominantemente idosos acima de } 60 \text { anos de idade. A LMA apresenta oito subtipos distintos mor- } \\
\text { fologicamente: LMA M0 a M7. Os métodos diagnósticos para identificação da LMA e classificação dos } \\
\text { subtipos são baseados em critérios morfológicos, citoquímicos e de imunofenotipagem, acrescidos de } \\
\text { análise genética. Além de ser importante para a diferenciação do tipo da linhagem da leucemia, se } \\
\text { mielóide (LMA) ou linfóide (LLA), o diagnóstico é também de grande importância para identificar a } \\
\text { leucemia bifenotípica aguda (BAL). O objetivo deste trabalho foi realizar uma revisão bibliográfica sobre } \\
\text { LMA, dando ênfase aos métodos laboratoriais utilizados para a sua identificação e diferenciação. }\end{array}$ \\
\hline
\end{tabular}

abstract

The acute leukemias are characterized by the clonal proliferation and maturative blockage of hematopoietic cells, with diffuse substitution of the bone marrow by neoplasic cells. The acute myeloid leukemia (AML) is a heterogeneous group of clonal disease in the hematopoietic tissue and predominantly affects people older than 60. The AML has eight morphologically different subtypes: AML M0 to M7. The diagnostic methods for identification of AML and subtypes classification are based on morphological, cytochemical and immunophenotyping patterns, besides genetic and molecular analyses. The diagnosis of leukemia is important to the lineage differentiation in AML or ALL and also for the identification of biphenotypic acute leukemia (BAL). The aim of this study was to perform a bibliographic review of AML, giving emphasis on laboratory methods useful for its identification and differentiation. key words

Acute myeloid leukemia

Cytochemistry

Immunophenotype

Cytogenetics

Molecular genetics.

\section{Introdução}

A leucemia é uma proliferação neoplásica generalizada ou acúmulo de células hematopoéticas, com ou sem envolvimento do sangue periférico ${ }^{(22)}$. Na maioria dos casos, as células leucêmicas extravasam para o sangue, onde podem ser vistas em grande número. Essas células também podem infiltrar o fígado, baço, linfonodos e outros tecidos ${ }^{(43)}$.
As leucemias são classificadas de acordo com o tipo celular envolvido e o grau de maturação das células. As leucemias agudas (LA) caracterizam-se pela proliferação clonal acompanhada de bloqueio maturativo (anaplasia) variável, o que possibilita a existência de diferentes subtipos de leucemias.

Nas leucemias agudas enquadram-se as leucemias mielóides agudas (LMA) ${ }^{(1)}$. A LMA é um grupo hete-

1. Graduanda em Biomedicina, Centro Universitário Feevale - RS.

2. Farmacêutico-Bioquímico do Weinmann Laboratório.

3. Professora-mestre da Faculdade de Farmácia da Universidade Federal do Rio Grande do Sul (UFRCS).

4. Professora-mestre do Curso de Biomedicina do Centro Universitário Feevale - RS. 
rogêneo de doenças clonais do tecido hematopoético, que se caracteriza pela proliferação anormal de células progenitoras da linhagem mielóide (mieloblastos), ocasionando produção insuficiente de células sangüíneas maduras normais, com conseqüente substituição do tecido normal. Desse modo, a infiltração da medula é freqüentemente acompanhada de neutropenia, anemia

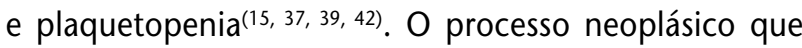
dá origem ao clone leucêmico pode surgir em qualquer estágio do desenvolvimento celular, ou seja, em qualquer fase da hematopoese ${ }^{(38)}$.

Os eventos moleculares precisos e responsáveis pela transformação leucêmica ainda não são conhecidos, entretanto o resultado final consiste na proliferação inexorável das células hematopoéticas imaturas que perderam a sua capacidade de diferenciação normal ${ }^{(17)}$. No entanto, a ativação de protoncogenes e as mutações (alterações genéticas), em genes supressores que regulam o ciclo celular, parecem estar envolvidas na patogênese das leucemias, pois levam à perda dos mecanismos normais controladores da proliferação (divisão celular), diferenciação-maturação e/ou da morte celular programada (apoptose) $)^{(4,39)}$.

Na maioria dos casos, a LA surge sem motivo aparente, embora algumas vezes possam se identificar possíveis causas, como:

- radiação ionizante;

- vírus oncogênicos (human T-lymphotropic virus type I [HTLV-I] causando leucemia das células T do adulto);

- fatores genéticos e congênitos;

- algumas substâncias químicas e fármacos (como benzeno e agentes alquilantes);

- predisposição a doenças hematológicas(13,35).

A LMA é uma doença predominante em adultos mais velhos (acima de 60 anos de idade), com mais de 50\% dos $\operatorname{casos}^{(25,37,44)}$. É mais comum no sexo masculino do que no feminino ${ }^{(4,13)}$, representa cerca de $15 \%-20 \%$ das LA da infância e $80 \%$ das dos adultos ${ }^{(20,39)}$ e apresenta um prognóstico pobre, especialmente em pacientes idosos ${ }^{(7)}$.

Segundo o Instituto Nacional do Câncer (INCA), os índices de leucemias no Brasil mostram-se altos e estimam-se, para o ano de 2006, aproximadamente 9.500 novos casos. Muitas introspecções novas para o diagnóstico, patogênese, manifestação clínica, tratamento e prognóstico dos pacientes com LMA refletem a heterogeneidade da doença, uma vez que o prognóstico entre subgrupos de pacientes com
LMA pode variar consideravelmente ${ }^{(37,51)}$. Com base nesses dados, tornam-se de grande importância clínica os avanços laboratoriais ocorridos no diagnóstico das leucemias nos últimos anos.

\section{Classificação}

As leucemias são classificadas com base no tipo celular envolvido e no estado de maturidade das células leucêmicas. Assim, as LA caracterizam-se pela presença de células muito imaturas (denominadas blastos) e por evolução rapidamente fatal em pacientes não-tratados ${ }^{(35)}$.

Os primeiros sistemas de classificação das LA eram baseados somente em investigações citomorfológicas e citoquímicas. A morfologia ainda representa um modelo central, mas foi incorporada em sistemas de classificações atuais, como imunofenotipagem para um delineamento mais preciso da linhagem hematopoética, e estágio de diferenciação de leucemias em particular ${ }^{(13,50)}$. Na Tabela 1 são abordadas as principais classificações das leucemias agudas $(2,6,7,10,21,38,50)$.

De acordo com o sistema de classificação French-American-British (FAB), as LMA são ainda morfologicamente subclassificadas em oito tipos:

- M0 - LMA sem diferenciação morfológica;

- M1 - LMA com mínima diferenciação morfológica;

- M2 - LMA com diferenciação (componente monocítico $<20 \%$;

- M3 - LMA promielocítica hipergranular

M3 variante hipogranular;

- M4 - LMA mielomonocítica (células monocíticas $\geq 20 \%$ ) M4 variante;

- M5 - LMA monocítica (com células monocíticas $\geq 20 \%$ das células leucêmicas)

M5a - LMA monoblástica (sem diferenciação, blastos $\geq 80 \%$ )

M5b - LMA monocítica (com diferenciação, blastos $<80 \%)$;

- M6 - eritroleucemia e variante;

- M7 - LMA megacarioblástica ${ }^{(26,38,55)}$.

Contudo, a classificação $F A B$ está em desuso e está sendo substituída pela classificação da Organização Mundial da Saúde (OMS). A classificação da OMS apresenta recomendações atualizadas e modificadas dos critérios diagnósticos utilizados pela $\mathrm{FAB}$, valorizando critérios citogenéticos ${ }^{(9)}$. 


\section{Tabela 1 Sistemas de classificação de leucemias agudas}

\begin{tabular}{ll}
\hline Grupo & Classificação \\
FAB $^{*}$ (1976) & Primeira classificação mundialmente aceita \\
& Critérios: morfologicos e citoquímicos \\
& Blastos na LA > 30\% na medula óssea \\
& LMA (M1 a M6) \\
& Em 1985, essa classificação foi revisada e adotaram-se critérios imunofenotípicos, incluindo o \\
& subtipo LMA M7, através da confirmação de blastos plaquetários, e o subtipo M0, através de \\
& marcadores monoclonais \\
& Critérios: morfológicos, imunológicos e citogenéticos \\
MIC** & Critérios: imunológicos (expressão de antígenos na superfície celular a partir de painéis de \\
EGIL*** (1995) & anticorpos monoclonais) \\
& Definição de leucemias bifenotípicas agudas (BAL) \\
& Foi proposta a classificação que separa e define os subtipos de leucemias agudas (mielóide, \\
& linfóide ou bifenotípica) \\
& Blastos na LA > 20\% na medula óssea \\
& LMA passa a ser valorizada através de seus dados de recorrência citogenética e da história \\
& clínica e/ou aspectos displásicos na medula óssea
\end{tabular}

*French-American-British.

**Morphological-Immunological-Cytogenetic.

***European Group for the Immunological Characterization of Leukemias.

****World Health Organization.

Adaptado de Szczepanski et al., 2003.

Existe também uma minoria de LA que apresenta características de ambas as linhagens (mielóide e linfóide) e, por essa razão, são designadas leucemias de linhagem mista, híbrida ou leucemia bifenotípica aguda (BAL) $)^{(10,40)}$. As BAL são doenças raras, responsáveis por mais de $5 \%$ das $\operatorname{LA}^{(3,7,32)}$.

\section{Diagnóstico laboratorial}

Geralmente, o diagnóstico de LMA inicia-se a partir de uma suspeita clínica (Tabela 2) e se baseia na avaliação do sangue periférico e da medula óssea ${ }^{(4,23,35)}$. Embora a morfologia continue sendo o fundamento para o diagnóstico, técnicas adicionais, incluindo imunofenotipagem, avaliação citogenética e estudos de genética molecular, tornaram-se essenciais e, em alguns casos específicos, são ferramentas complementares obrigatórias ${ }^{(49,51)}$. O uso de procedimentos diagnósticos permite a identificação do tipo celular envolvido na leucemogênese, o que é fundamental para orientar a terapêutica e determinar, até certo ponto, o prognóstico das leucemias ${ }^{(14)}$.

As colorações citoquímicas (Tabela 3) usadas no diagnóstico e na classificação das leucemias podem ser aplicadas tanto à medula óssea quanto ao sangue periférico, auxilian- do na confirmação da origem mielóide e/ou monocítica. Apesar dos progressos da imunofenotipagem, as reações citoquímicas ainda são úteis no diagnóstico das LMA.

As principais colorações em uso são fosfatase alcalina; mieloperoxidase (MPO); sudão negro B (sudan black $B$ [SBB]); naftol AS-D; cloroacetato esterase (CAE); esterases inespecíficas, como alfa-naftil acetato esterase (ANAE); reação do ácido para-aminossalicílico (ácido periódico de Schiff [PAS]) e fosfatase ácida.

A mieloperoxidase ou SBB positiva confirma a natureza mielóide dos blastos e revela os bastonetes de Auer em aproximadamente $65 \%$ dos casos. Ela é específica para as linhagens de granulócitos, eosinófilos e monóci-

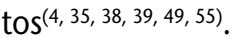

A imunofenotipagem é realizada por meio de anticorpos monoclonais marcados, que reconhecem epítopos específicos de antígenos celulares. As técnicas empregadas na imunofenotipagem podem ser a citometria de fluxo ou a imunocitoquímica. A importância da imunofenotipagem reside, principalmente, no diagnóstico das LMA M0 e M7, mas também em alguns casos de M5a, além de auxiliar no diagnóstico das LMA M3, LMA M2 e LMA M1/M2, bem

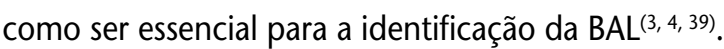




\section{Tabela 2 Características clínicas das IMA}

\begin{tabular}{|c|c|}
\hline Suspeita clínica & $\begin{array}{l}\text { - palidez } \\
\text { - hepatomegalia } \\
\text { - esplenomegalia } \\
\text { - linfadenopatia } \\
\text { - febre em consequiência de infecções } \\
\text { - faringite } \\
\text { - petéquias e outras manifestações hemorrágicas } \\
\text { - dor óssea } \\
\text { - hipertrofia gengival } \\
\text { - infiltrações cutâneas }\end{array}$ \\
\hline Hemograma & $\begin{array}{l}\text { - contagem de plaquetas e hemoglobinas baixas } \\
\text { - contagem de células brancas pode variar de }<1.000 / \mu \mathrm{l} \text { a } 200.000 / \mu \mathrm{l} \\
\text { - contagem diferencial de células brancas anormais com neutropenia e presença de } \\
\text { blastos; geralmente, detecção pelos flags em aparelho } \\
\text { - anemias normocrômica e normocítica; trombocitopenia pode ser severa }\end{array}$ \\
\hline
\end{tabular}

Adaptado de Bain, 2003.

\section{Tabela 3 Colorações citoquímicas}

\begin{tabular}{|c|c|}
\hline Mo & $\mathrm{MPO}^{-} ; \mathrm{SBB}^{-}$; esterases \\
\hline M1 & $\mathrm{MPO} / \mathrm{SBB}^{+}$em $\geq 3 \%$ das células \\
\hline M2 & $\mathrm{MPO}^{+} ; \mathrm{SBB}^{+}$ \\
\hline M3 & $\mathrm{MPO}^{++} ; \mathrm{SBB}^{++}$ \\
\hline M4 & $\mathrm{MPO}^{+}$; $\mathrm{SBB}^{+}$; esterase inespecífica ${ }^{+}$com inibição pelo $\mathrm{NaF}^{-} \mathrm{ANAE}^{+}$ \\
\hline M5 & MPO; esterase $^{++}$ \\
\hline M6 & $\mathrm{MPO} ; \mathrm{SBB}^{-} ; \mathrm{ANAE}^{++}$ \\
\hline M7 & $\mathrm{MPO}^{-} ; \mathrm{SBB}^{-} ; \mathrm{ANAE}^{+}$na zona de Golgi \\
\hline
\end{tabular}

MPO: mieloperoxidase; SBB: sudão negro B (Sudan black B); ANAE: alfa-naftil acetatoesterase; NaF: fluoreto de sódio; (+): positivo; (-): negativo. Adaptado de Yamoto, 2000.

A citometria de fluxo é realizada com maior freqüência para distinção entre as leucemias mielóide e linfóide, o que é de crucial importância, e na monitorização pós-tratamento da LMA para detecção de doença residual mínima ${ }^{(45,49,54,57)}$. Também é útil na correta identificação dos subtipos de LMA, como a LMA M0, e na diferenciação da leucemia promielocítica aguda (M3) da LMA M1/M2 ${ }^{(8,27)}$. Geralmente, a imunofenotipagem é realizada em suspensões de células do sangue periférico e da medula óssea, mas, quando necessário, pode ser feita em cortes histológicos ${ }^{(4)}$. A Tabela 4 resume os principais marcadores imunofenotípicos relacionados aos subtipos de $\operatorname{LM}(33,49,50)$.

A citogenética e os estudos moleculares freqüentemente detectam anormalidades dentro do clone leucêmico, podendo sugerir o diagnóstico e/ou o prognóstico ${ }^{(20,35,49)}$. As aberrações citogenéticas adquiridas são detectadas em 55\%-75\% de pacientes recentemente diagnosticados com
$\operatorname{LMA}^{(18,36,37,53)}$. A análise citogenética é convencionalmente feita pela análise microscópica dos cromossomos das células da medula óssea ${ }^{(4)}$ durante a metáfase ${ }^{(19,49)}$. Pode ser complementada por técnicas de hibridização in situ, particularmente a hibridização fluorescente in situ (FISH), sendo importante para confirmar a presença de rearranjos recorrentes ${ }^{(28,29)}$. Atualmente, a análise citogenética tem sido considerada um instrumento diagnóstico de grande importância para determinação do fator prognóstico da $\operatorname{LMA}^{(19,37,46,56) \text {. }}$

A análise de genética molecular pode se fundamentar na análise do ácido desoxirribonucléico (DNA) por meio de técnicas, como análise de Southern blot ou reação em cadeia da polimerase (PCR), ou na análise do ácido ribonucléico (RNA) por PCR da transcriptase reversa (RT-PCR) e também por PCR em tempo real (RQ-PCR). A finalidade da análise de genética molecular pode ser tanto o estabelecimento da 
clonalidade, pela detecção da recombinação de genes que expressam imunoglobulinas, quanto pela identificação de uma recombinação molecular característica de um determinado tipo de LMA. Além de auxiliar na classificação das $L A$, é útil no monitoramento da doença residual mínima

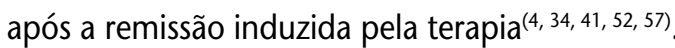

A importância da citogenética e da genética molecular na LMA para classificação e para compreensão de mecanismos patogênicos é apreciada cada vez mais no contexto clínico ${ }^{(16,19)}$. As aberrações citogenéticas mais comuns observadas na LMA, e suas freqüências, estão listadas na

Tabela $5^{(5,11,12,18,31,46,47)}$.

\section{Tabela 4 Classificação da imunofenotipagem da LMA}

\begin{tabular}{lccccc}
\hline Marcadores & LMA-M0/M1/M2 & LMA-M3 & LMA-M4/M5a/M5b & LMA-M6 & LMA-M7 \\
CD13/CD33 & ++ & ++ & ++ & + & ++ \\
CD65 & $\pm /+/+$ & + & ++ & \pm & \pm \\
MP0 & $-/+/++$ & ++ & ++ & + & - \\
CD11c & - ou \pm & - & ++ & - & - \\
CD14 & - & - & $+/+/+$ & - & - \\
CD15 & $\pm / \pm /++$ & \pm & - & - & - \\
CD36 & - & - & + & ++ & + \\
H-antígeno & - & - & - & ++ & + \\
CD235a & & - & - & & \\
(Glicoforina A) & - & - & - & + & - \\
CD41/CD61 & - & - & - & - & ++ \\
CD42 & - & \pm & $++/ \pm$ & + & + \\
CD34 & $++++/+$ & + & + & + & ++ \\
CD117 & ++ & + & ++ & + & + \\
HLA-DR & ++++++ & - & + & + & ++ \\
TdT & + & \pm & + & + \\
\hline
\end{tabular}

$-:<10 \%$ das leucemias são positivas; \pm : 10\%-25\% das leucemias são positivas; $+: 25 \%-75 \%$ das leucemias são positivas; $++:>75 \%$ das leucemias são positivas. Adaptado de Szczepanski et al., 2003.

\section{Tabela 5 Análise citogenética: cromossomos anormais na LMA}

\begin{tabular}{|c|c|c|c|c|}
\hline \multirow[b]{2}{*}{ Anormalidades } & \multirow[b]{2}{*}{ Fusão de genes } & \multirow[b]{2}{*}{ Subtipo } & \multicolumn{2}{|c|}{ Frequiência } \\
\hline & & & Crianças (\%) & Adultos (\%) \\
\hline $\mathrm{t}(8 ; 21)(\mathrm{q} 22 ; \mathrm{q} 22)$ & AMLI-ETO & M2/M1 & $10-15$ & $8-12$ \\
\hline $\operatorname{inv}(16)(p 13 q 22)$ & CBF $\beta$-MYHII & M4eo & $6-12$ & $8-12$ \\
\hline $\mathrm{t}(15 ; 17)(\mathrm{q} 22 ; \mathrm{q} 21)$ & PML-RAR $\alpha$ & M3/M3v & $8-15$ & $8-10$ \\
\hline $\mathrm{t}(9 ; 11)(\mathrm{p} 22 ; \mathrm{q} 23)$ & MLL-AF9 & M5a & $8-10$ & $1-2$ \\
\hline $\mathrm{t}(3 ; 21)(\mathrm{q} 26 ; \mathrm{q} 22)$ & AMLI-EAP/EVII & - & 1 & $<1$ \\
\hline $\mathrm{t}(6 ; 9)(\mathrm{p} 23 ; \mathrm{q} 34)$ & DEK-CAN & M1/M2 & $1-2$ & Rara \\
\hline $\operatorname{inv}(3)(q 21 ; q 26)$ & EVII & - & $<1$ & $1-2$ \\
\hline $\mathrm{t}(1 ; 22)(\mathrm{p} 13 \mathrm{q} 13)$ & OTT-MAL & M7 & 2 & $<1$ \\
\hline Trissomia do 8 & & - & $1-4$ & $3-5$ \\
\hline Trissomia do 11 & & M1/M2 & - & $<1$ \\
\hline Complexo & & - & 6 & $10-20$ \\
\hline
\end{tabular}

Adaptado de Schoch \& Haferlach, 2002. 


\section{Discussão e conclusão}

Até bem pouco tempo, o diagnóstico da LMA era baseado exclusivamente na morfologia e na citoquímica do sangue e da medula óssea. Atualmente, o desenvolvimento de anticorpos monoclonais e citometria de fluxo assumiram o papel principal para a definição precisa das células blásticas de linhagem mielóide e subtipos de $\operatorname{LMA}^{(49)}$.

A classificação das LA permite a correta separação dos pacientes leucêmicos nas categorias LMA e LLA ${ }^{(4)}$. Entretanto, foi a partir dos sistemas de classificação, juntamente com os avanços ocorridos em imunofenotipagem, e devido a crescente importância em eventos genéticos, que hoje há o reconhecimento da importância de um diagnóstico mais preciso, indicando fatores prognósticos e uma terapia mais adequada a cada subtipo de $\operatorname{LMA}^{(10,50)}$.

A identificação de fatores prognósticos permite a estratificação dos pacientes em grupo de risco, não generalizando os subtipos de LMA, o que possibilita uma abordagem terapêutica diferenciada. Além disso, os métodos diagnósticos para uma avaliação adequada somente serão possíveis com estudos mais aprofundados das técnicas, baseados em uma ampla combinação dos mesmos. Porém, esses estudos são caros, consomem tempo e requerem habilidade de pessoas experientes em laboratórios referenciais centralizados $^{(14,30)}$.

Experiências clínicas têm demonstrado a importância vital de anormalidades citogenéticas na determinação da sobrevida em pacientes com LMA. Aprimoramentos em tecnologias moleculares também estão em foco na detecção de translocações clinicamente relevantes, bem como providências de métodos sensíveis para rastrear doença residual mínima, em muitos pacientes, e para descrever a caracterização da LMA no diagnóstico e/ou durante o tratamento(13,31, 49).

Sabe-se que o grau de evolução da LMA está significativamente relacionado ao aumento da proliferação celular clonal. Assim, a partir da análise genética, a expressão dos genes identificados na doença fornece razões para estudos futuramente preditivos para diagnóstico e intervenção terapêutica ${ }^{(48)}$.

\section{Referências}

I. ANJOS, A.R.; SILVA, M.A.; BORELLI, P. Matriz extracelular e leucemia. Rev Bras Hematol Hemoter, v. 22, n. 3, p. 404 12, 2000.

2. BAIN, B.J. Classification of acute leukaemia: the need to incorporate cytogenetic and molecular genetic information. J Clin Pathol, v. 5I, n. 6, p. 420-3, 1998.

3. BAIN, B.J. et al. Revised guideline on immunophenotyping in acute leukaemias and chronic lymphoproliferative disorders. Clin Lab Haem, v. 24, p. I- I 3, 2002.

4. BAIN, J.B. Diagnóstico em Leucemias. 2. ed. Rio de Janeiro: Revinter, 2003.

5. BENCH, A.J.; ERBER, W.N.; SCOTT, M.A. Molecular genetic analysis of haematological malignancies: I. Acute leukaemias and myeloproliferative disorders. Clin Lab Haem, v. 27, p. |48-7|, 2005

6. BENE, M.C. et al. Proposals for the immunological classification of acute leukemias: European Group for the Immunological Characterization of Leukemias (EGIL). Leukemia, v. 9, p. 1783-6, 1995

7. BENE, M.C. et al. Impact of immunophenotyping on management of acute leukemias. Haematologica, v. 84, n. I I, p. 1024-34, 1999.

8. BROWN, M.; WITTEWER, C. Flow cytomety: principles and clinical applications in hematology. Clin Chem, v. 46, n. 8(B), p. $1221-9,2000$.

9. BRUCE, D.C. et al. Revised Recommendations of the Internacional Working Group for Diagnosis, Standardization of Response Criteria,Treatment Outcomes, and Reporting Standards for Therapeutic Trials in Acute Myeloid Leukemia.J Clin Oncol, v. 2 I, n. 24, p. 4642-9, 2003.

I0. BRUNNING, R.D. Classification of acute leukemias. Semin Diagn Pathol, v. 20, n. 3, p. I42-53, 2003.

I I. CHAUFFAILLE, M.L.L.F.; BORRI, D.; MARTINS, S.L.R. Leucemia mielóide aguda $t(8 ; 21)$ : freqüência em pacientes brasileiros. Rev Bras Hematol Hemoter, v. 26, n. 2, p. 99- I03, 2004.

12. DASH, A.; GILLILAND, D.G. Molecular genetics of acute myeloid leukaemia. Best Pract Res Clin Haematol, v. I4, n I, p. 49-64, 2001.

13. DOUER, D.The epidemiology of acute promyelocytic leukaemia. Best Pract Res Clin Haematol, v. I6, n. 3, p. 357-67, 2003.

14. FARIAS, M.G.; CASTRO, S.M. Diagnóstico laboratorial das leucemias linfóides agudas. J Bras Patol Med Lab, v. 40, n. 2, p. 9 I-8, 2004

15. FERRARA, F. Unanswered questions in acute myeloid leukaemia Lancet Oncol, v. 5, p. 443-50, 2004. Disponível em: http:// oncology.thelancet.com. Acesso em: 20 nov. 2004. 
16. GILES, F.J. et al. Acute myeloid leukemia. Hematology, p. 731 10, 2002.

17. GOLDMAN, L:; BENNETT,J.C. CecilTratado de Medicina Interna. 21 ed. São Paulo: Guanabara Koogan, 2001.

18. GRIMWADE, D. The clinical significance of cytogenetic abnormalities in acute myeloid leukaemia. Best Pract Res Clin Haematol, v. I4, n. 3, p. 497-529, 200 I.

19. HAFERLACH, T. et al. Genetic classification of acute myeloid leukemia (AML). Ann Hematol, v. 83, n. I, p. 97-100, 2004.

20. HALL, G.W. Childhood myeloid leukaemias. Best Pract Res Clin Haematol, v. 14, n. 3, p. 573-91, 2001.

2।. HARRIS, N.L. et al.World Health Organization Classification of Neoplasic Diseases of The Hematopoietic and Lymphoid Tissues: report of the clinical advisory committee meeting. J Clin Pathol, v. 17, p. 3835-49, 1999.

22. HENRY, J.B. Diagnósticos Clínicos e Tratamento por Métodos Laboratoriais. 2 ed. São Paulo: Manole, 1999.

23. HOYER, J.D. et al. Detection and classification of acute leukemia by the Coulter STKS Hematology Analyzer. Am J Clin Pathol, v. I06, n. 3, p. 352-8, 1996.

24. INSTITUTO NACIONAL DO CÂNCER (INCA). Disponível em http://www.inca.gov.br/estimativas/2003/. Acesso em: 30/jul/2005.

25. IOVINO, C.S.; CAMACHO, L.H. Acute myeloid leukemia: a classification and treatment update. Clin J Oncol Nurs, v. 7, n. 5, p. 535-40, 2003.

26. JENNINGS, C.D.; FOON, K.A. Recent advances in flow cytometry: application to the diagnosis of hematologic malignancy. Blood, v. 90, n. 8, p. 2863-92, 1997.

27. KALEEM, Z. et al. Flow cytometric analysis of acute leukemias: diagnostic utility and critical analysis of data. Arch Pathol Lab Med, v. 127, p. 42-8, 2003.

28. KANG, L.C. et al. Two cases of acute myeloid leukemia with $t(1|;| 7)$ associated with varying morphology and immunophenotype: rearrangement of the MLL gene and a region proximal to the RARalpha gene. Cancer Genet Cytogenet, v. 159, n. 2, p. 168-73, 2005.

29. KEARNEY, L. Molecular cytogenetics. Best Pract Res Clin Haematol, v. I4, n. 3, p. 645-68, 2001.

30. KERN, W. et al. Gene expression profiling as a diagnostic tool in acute myeloid leukemia. Am J Pharmacogenomics, v .4, n. 4, p. 225-37, 2004.

3।. LANGMUIR, P.B.; APLENC, R.; LANGE, B.J. Acute myeloid leukaemia in children. Best Pract Res Clin Haematol, v. I4, n. I, p. 77-93, 2001.

32. LEE, P.S. et al. Acute leukemia with myeloid, B-, and natural killer cell differentiation. Arch Pathol Lab Med, v. 127, n. 2, p. 93-5, 2003.

33. LEFTEROVA, P; SHIMIDT-WOLF, I.G.H. Coexpression of lymphoid and myeloid markers on cell surfaces. Leuk Lymphoma, v. 26, p. 27-33, 1996.

34. LEROY, H. et al. Prognostic value of real-time quantitative
PCR (RQ-PCR) in AML with t(8;21). Leukemia, v. 19, n. 3, p. 367-72, 2005.

35. LIESNER, R.J;; GOLDSTONE,A. H.ABC of clinical haematology: the acute leukemias. Br J Haematol, n. 314, p. 733, 1997.

36. LINDVALL, C. et al. Combined genetic and transcriptional profiling of acute myeloid leukemia with normal and complex karyotypes. Haematologica, v. 89, p. 1072-81, 2004.

37. LÖWENBERG, B. Prognostic factors in acute myeloid leukaemia. Best Pract Res Clin Haematol, v. I4, n. I, p. 65-75, 2001.

38. LUSIS, M.K.P. Classificação FAB das leucemias mielóides agudas. Rev Bras Hematol Hemoter, v. 22, n. 2, p. I 175-8, 2000.

39. MARTINS, S.L.R.; FALCÃO, R.T. A importância da imunofenotipagem na leucemia mielóide aguda. Rev Assoc Med Bras, v. 46, n. I, p. 57-62, 2000.

40. MATUTES, E. et al. Definition of acute biphenotypic leukemia. Haematologica, n. 82, p. 64-6, 1997.

4I. MRÓZEK, K.; HEINONEN, K.; BLOOMFIELD, C.D. Clinical importance of cytogenetics in acute myeloid leukaemia. Best Pract Res Clin Haematol, v. 14, n. I, p. 19-47, 2001.

42. PELLOSO, L.A.F. et al. Cariótipo em leucemia mielóide aguda: importância e tipo de alteração em 30 pacientes ao diagnóstico. Rev Assoc Med Bras, v. 49, n. 2, p. I50-5, 2003.

43. ROBBINS, S.L. et al. Patologia Estrutural e Funcional. 5 ed. Rio de Janeiro: Guanabara Koogan, 1996.

44. RODRIGUES, C.A. et al. Acute myeloid leukemia in elderly patients: experience of a single center. Braz J Med Biol Res, v. 36, n. 6, p. 703-8, 2003.

45. SAN-MIGUEL, J.F.;VIDRIALES, M.B.; ORFÃO,A. Immunological evaluation of minimal residual disease (MRD) in acute myeloid leukaemia (AML). Best Pract Res Clin Haematol, v. 15, n. I, p. 105- 18, 2002.

46. SCHOCH, C.; HAFERLACH,T. Cytogenetics in acute myeloid leukemia. Curr Oncol Rep, v. 4, p. 390-7, 2002.

47. $\mathrm{SCHOCH}, \mathrm{C}$. et al. Acute myeloid leukemia with a complex aberrant karyotype is a distinct biological entity characterized by genomic imbalances and a specific gene expression profile. Genes Chromosomes Cancer, v. 43, n. 3, p. 227-38, 2005.

48. STABER, P.B. et al. Common alterations in gene expression and increased proliferation in recurrent acute myeloid leukemia. Oncogene, v. 23, n. 4, p. 894-904, 2004.

49. SWIRSKY, D.M.; RICHARDS, S.J. Laboratory diagnosis of acute myeloid leukaemia. Best Pract Res Clin Haematol, v. I4, n. I, p. I- 17, 2001.

50. SZCZEPANSKI, T.; van VELDEN, V.H.J.; van DONGEN, J.J.M. Classification systems for acute and chronic leukemias. Best Pract Res Clin Haematol, v. 16, n. 4, p. 56I-82, 2003.

5I.TALLMAN, M.S. Relevance of pathologic classifications and diagnosis of acute myeloid leukemia to clinical trials and clinical practice. Cancer Treat Res, n. 121, p. 45-67, 2004.

52. TAYLOR, C.G. et al. Diagnosis and classification of the acute 
leukemias: recent advances and controversial issues. Hematopathol Mol Hematol, v. I0, n. I-2, p. I-38, 1996.

53.WEIR, E.G.; BOROWITZ, M.J. Flow cytometry in the diagnosis of acute leukemia. Semin Hematol, v. 38, n. 2, p. 124-38, 2001.

54. WANG, X.B. et al. Correlation of immunophenotype to cytogenetics and clinical features of adult acute myeloid leukemia. Ai Zheng, v. 24, n. 6, p. 667-7I, 2005.

55. YAMAMOTO, M. Imunofenotipagem em leucemias mielóides agudas. Rev Bras Hematol Hemoter, v. 22, n. 2, p. 169-74, 2000.

56. YANADA, M. et al. Efficacy of allogenic hematopoietic stem cell transplantation depends on cytogenetic risk for acute myeloid leukemia in first disease remission: a metaanalysis. Cancer, v. 103, n. 8, p. 1652-8, 2005.

57. YIN, J.A.L. Minimal residual disease in acute myeloid leukaemia. Best Pract Res Clin Haematol, v. I 5, n. I, p. I 19-35, 2002. 